\section{Asthma bei Kindern - Compliance entscheidet über Therapie-Erfolg}

A sthma bei Kindern stellt immer noch eine diagnostische und therapeutische Herausforderung dar, so J. O. Warner, Southampton. Während früher „kindliches Asthma“ zu selten diagnostiziert wurde, geschieht dies heute eher zu häufig. So ist ein gelegentlich auftretender Husten ohne weitere Begleitsymptome sicher nicht als Asthma zu werten. Auch rezidivierende virale Infekte bei Kleinkindern werden mittlerweile zu oft als Asthma fehldiagnostiziert.

Unbestritten hat jedoch die Asthmainzidenz bei Kindern zugenommen. $\mathrm{Ab}$ dem Schweregrad 2 werden häufig topische Steroide eingesetzt. Seit langem bekannt sind jedoch die Compliance-Probleme bei der Anwendung sowohl von DNCG als auch von lokalen Steroiden, die den Erfolg der regelmäßig durchzuführenden Inhalationsbehandlung schmälern können. Dafür spricht nach den Worten Warners u. a. die nach wie vor hohe Morbidität der Kinder. Vor allem Schlafstörungen und damit verbundene Leistungsminderung am Tage sind häufige Klagen der Eltern.

Kompliziert wird die Situation dadurch, dass etwa 60 bis $80 \%$ der Asthmakinder auch eine allergische Rhinitis haben, $50 \%$ von ihnen ein atopisches Ekzem. Das bedeutet eine zusätzliche Belastung, da dadurch bis zu drei unterschiedliche lokale Therapieformen benötigt werden. Die pragmatische Lösung dieser Probleme liegt in der Vereinfachung der Therapie, sagte Warner. Dies ist heute durch die orale Applikation von Leukotrien-Rezeptor-Antagonisten möglich. Sie wirken bei Asthma und Rhinitis und möglicherweise auch bei atopischer Dermatitis. Die Verträglichkeit ist sehr gut, bereits Kinder $a b$ zwei Jahren können behandelt werden.

Studien belegen die gute Wirksamkeit von Leukotrien-Rezeptor-Antagonisten wie Montelukast bei Asthma oder Rhinitis, erklärte T. Sampson, Southampton, Großbritannien. Denn die Leukotriene, speziell die Cysteinyl-Leukotriene (Cys-LTs), haben einen entscheidenden Anteil an der Symptomatik von
Asthma und Rhinitis: Sie sind potente Mediatoren von Bronchokonstriktion und Mukushypersekretion, sie verschlechtern die mukoziliare Clearance und sind mit verantwortlich für die Ausbildung von vaskulären Ödemen, bronchialer Hyperreaktivität und sie locken Entzündungszellen in das Gewebe. Bei bronchialer oder nasaler Allergenprovokation finden sich erhöhte Cys-LT-Spiegel in der bronchialen bzw. nasalen Lavage.

Speziell für Kinder werden zwei orale Dosierungsformen von Montelukast angeboten: Die 4-mg-Einzeldosis (Sin- gulair $^{\circledR}$ mini) für die Behandlung von Kleinkindern ab zwei Jahren und die 5mg-Einzeldosis (Singulair junior ${ }^{\circledR}$ ) für Kinder ab sechs Jahren. Offiziell zugelassen ist das Präparat in Deutschland zur Monotherapie des Belastungsasthmas sowie für die Kombinationsbehandlung mit Kortikoiden in der Dauertherapie.

Doch entscheidend für den Therapieerfolg eines Medikaments, so J. Kemp, San Diego, Kalifornien, ist nicht allein seine belegte Wirksamkeit in klinischen Studien, sondern vor allem seine Akzeptanz durch die Kinder. $\quad b k$

\section{Satellitensymposium „Evolving Role of LTRAs in the Management of Pediatric Asthma \& Allergy“ anlässlich des Inter- national Pediatric Respiratory and Allergy Congress, Prag (Tschechische Republik),}

\title{
Vom Diskus zum Dosieraerosol
}

$M$ it einer neuen Darreichungsform von atmadisc $^{\circledR}$ erweitert die Schwarz Pharma Deutschland GmbH das Produkt-Portfolio im AsthmaBereich: atmadisc ${ }^{\circledR}$, eine Fixkombination mit den bewährten Substanzen Fluticason und Salmeterol, ist seit Mai nun auch als FCKW-freies Dosieraerosol erhältlich. Das Monheimer Pharmaunternehmen, das atmadisc ${ }^{\circledR}$ mit dem Applikationssystem Diskus ${ }^{\circledR}$ bereits im September 2000 einführte, bietet nun für praktisch jeden Asthmatiker das passende Inhalationsgerät an. Das atmadisc ${ }^{\circledR}$ Dosier-Aerosol ist in drei Wirkstärken erhältlich: Jeweils $25 \mu \mathrm{g}$ Salmeterol werden mit $50 \mu \mathrm{g}, 125$ $\mu \mathrm{g}$ oder $250 \mu \mathrm{g}$ Fluticason kombiniert. Die Kombination von Salmeterol und Fluticason ist die einzige fixe Kombination dieser Substanzklassen, die als Dosieraerosol verfügbar ist.

Das atmadisc ${ }^{\circledR}$ Dosieraerosol ist für die Patienten geeignet, die mit der Handhabung eines Dosieraerosols bereits bestens vertraut sind und daher eine Umstellung auf ein anderes Inhalationssystem scheuen. Bei Asthmatikern, die zur Bronchodilatation und Entzündungshemmung bislang zwei Dosieraerosole anwenden mussten, lässt die Therapievereinfachung mit atmadisc ${ }^{\circledR}$ eine bessere Compliance erwarten. Überdies ermögli- chen die synergistischen Effekte der Kombination beider Wirkdtoffe eine ausgezeichnete Asthma-Kontrolle. Vorteilhaft ist auch der Preis, der günstiger ist als bei den freien Kombinationen.

atmadisc ${ }^{\circledR}$ ist für die regelmäßige Behandlung des Asthma bronchiale zugelassen und entspricht den aktuellsten Empfehlungen der Deutschen Atemwegsliga. atmadisc ${ }^{\circledR}$ vereint die beiden Säulen der Astmatherapie in einem Medikament: Fluticason zur Entzündungshemmung und Salmeterol zur Bronchodilatation. Die Vorteile der fixen Kombination sind evident. Bei nur zweimal täglicher Anwendung können die Häufigkeit der Exazerbationen und die Lebensqualität signifikant günstig beeinflusst werden. Die Compliance des Patienten kann gesteigert werden, da lediglich ein Inhalator angewendet werden muss. Das innovative Pulver-Inhalationssystem Diskus ${ }^{\circledR}$ ermöglicht neben einer sehr guten Wirkstoffverteilung auch eine einfache Handhabung. Mit dem Dosieraerosol steht atmadisc ${ }^{\circledR}$ nun auch in einer bewährten Applikationsform zur Verfügung, die die Palette von Schwarz Pharma sinnvoll ergänzt.

Nach Informationen von Schwarz Pharma Deutschland 